\title{
Probing nanoscale potential modulation by defect-induced gap states on GaAs(110) with light-modulated scanning tunneling spectroscopy
}

\author{
Shoji Yoshida, Yuya Kanitani, Osamu Takeuchi, and Hidemi Shigekawa ${ }^{\text {a) }}$ \\ Institute of Applied Physics, CREST-JST, University of Tsukuba, Tsukuba 305-8573, Japan
}

(Received 6 October 2007; accepted 5 February 2008; published online 12 March 2008)

\begin{abstract}
We investigated charged defects on an $n$-GaAs(110) surface using light-modulated scanning tunneling spectroscopy. Tunneling via a single defect-induced gap state under photoillumination was observed for the isolated atomic defects. Screened Coulomb potentials induced around a charged $\mathrm{Ga}$ vacancy and a step edge were visualized, for the first time, with a nanometer spatial resolution. Furthermore, the charge states of the individual defects were determined on the atomic level. (C) 2008 American Institute of Physics. [DOI: 10.1063/1.2888733]
\end{abstract}

In the history of the development of functional materials and devices, defects, which disturb the electronic and optical properties, were considered a problem to be avoided. However, recent progress in nanoscale science and technology has drastically changed the entire situation through the introduction of a new concept; "defect engineering"- defects are actively designed and controlled to realize desired functions. The current reduction in the size of semiconductor devices has intensified the urgency of the requirement and made the atomic scale characterization of defects and impurities unprecedentedly important. ${ }^{1}$

Scanning probe microscopy is a powerful technique because of its high spatial resolution. Since the development of scanning tunneling microscopy (STM), various techniques based on STM have been employed to analyze the atomic structures and electronic states of defects and impurities on semiconductor surfaces. ${ }^{2-8}$ Defects in semiconductors are electrically charged in general and affect the electronic properties of semiconductors through, for example, the Fermi level pinning and compensation of dopants. 3,4 Therefore, considerable efforts have been focused on determining the charge states of various types of defects by using STM.,6

The sign of the electronic charge of a defect can be deduced from the topographic contrast of a STM image, which depends on the relationship between the charge of the defect and the polarity of the tunneling bias voltage. ${ }^{7}$ The spatial distribution of the topographic contrast originates from the screened Coulomb potential (SCP), which is comparable to the Debye screening length of a sample. However, since an exact electrostatic potential cannot be obtained using STM, determining the exact charge state of a defect using STM is difficult. Therefore, indirect methods, such as measuring the average distance between defects that reflect Coulomb repulsion and measuring the number of charged defects that are coupled with the predetermined dopants, have been employed in pioneering works to determine the charge states of defects. ${ }^{3-6}$ For further advancement in functional materials and devices based on nanoscale science and technology, development of a method that enables the direct characterization of the surveyed individual defects is required.

In this study, we demonstrated a method that enables us to visualize the nanoscale electrostatic potential distribution

${ }^{a)}$ URL: http://dora.bk.tsukuba.ac.jp. induced by individual defects and to directly determine the charge states of these defects. We determined the charge states of Ga vacancies and [110] atomic steps from the results of the analysis of the SCPs visualized using the analysis of spatially resolved surface photovoltage (SPV). Tunneling under photoillumination via defect-induced gap states (DIGSs) is also discussed.

Around a charged defect, band bending is induced by the screening of the charge. The profile of the potential distribution shows the electronic charge trapped in the defect. Under laser illumination, photogenerated electron-hole pairs are separated by electric fields and redistributed to reduce band bending. This change in the surface potential of a semiconductor induced by super-band-gap illumination is the SPV, which provides information about the polarity and magnitude of surface band bending under dark condition. ${ }^{9,10}$ If a flat band condition is achieved with a sufficient laser intensity, a spatially resolved SPV directly exhibits a local electrostatic potential variation. However, the penetration of the bias voltage applied between a sample and a tip induces band bending [tip-induced band bending (TIBB)] below a STM tip. TIBB strongly depends on various parameters, such as tunneling bias voltage, tip-sample distance, and the doping level of the sample, resulting in difficulty in SPV measurement.

To accurately estimate the SPV, we employed lightmodulated scanning tunneling spectroscopy (LM-STS), which enables us to measure the tunneling current-bias voltage $(I-V)$ spectra at a fixed tip-sample distance under both dark and light conditions simultaneously. ${ }^{11,12}$ The SPV is calculated from the shift of the two $I-V$ spectra.

As a sample, an $n$-GaAs(110) surface ( $\mathrm{Si}$ doped, $2 \times 10^{18} \mathrm{~cm}^{-3}$ ) was prepared by cleavage in a vacuum $\left(<1 \times 10^{-8} \mathrm{~Pa}\right)$. Mechanically chopped illumination from a laser diode $(635 \mathrm{~nm}, 100 \mathrm{~Hz}), 60^{\circ}$ off normal to the surface, was focused onto the tunnel junction area with a spot diameter of $0.02 \mathrm{~mm}$. For a STM tip, an electrochemically etched tungsten tip was used.

Figure 1 shows the filled state topographic images of (a) a Ga vacancy and (b) a [110] step on a GaAs(110) surface. In Fig. 1(a), the Ga vacancy exhibits a contrast of a bright elevation at the center of the defect location, which is surrounded by a dark depression region. This is the signature of a negatively charged defect; ${ }^{7}$ a dangling bond of the Ga vacancy appears bright in the filled state image. The [110] step 


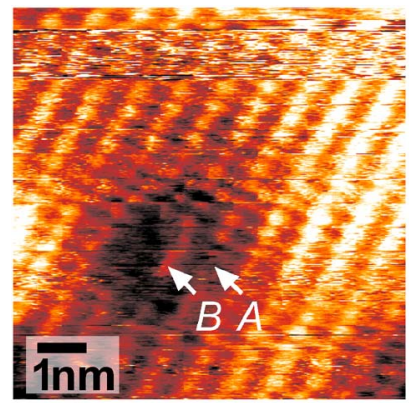

(a) (b)

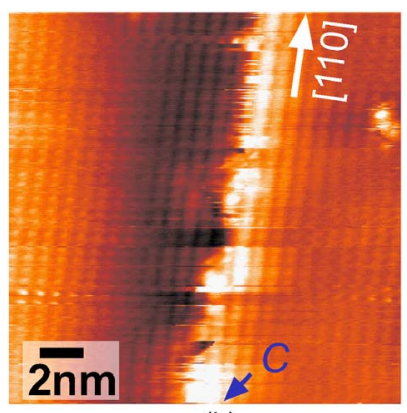

FIG. 1. (Color online) Filled state topographic images of (a) Ga vacancy and (b) [110] step on $\operatorname{GaAs}(110)$ surface.

shows a similar image consisting of a depression region along the bright step line.

Figure 2 shows the obtained tunneling current-bias voltage (I-V) spectra for (a) A and (b) B (Ga vacancy) in Fig. 1(a) and (c) C in Fig. 1(b) ([110] step). The tunneling junction has a metal-insulator-semiconductor (MIS) structure, as schematically illustrated in Fig. 2(d). Since the GaAs(110) surface has no surface state within the bulk band gap, TIBB easily occurs at positive sample bias voltages, while it scarcely occurs at negative bias voltages. ${ }^{12}$

A negative sample bias voltage of $-1.7 \mathrm{~V}$, at which no photocurrent was detected, was chosen as a set point for LM-STS measurements. In the positive-sample-bias-voltage region, the tunneling current oscillates owing to the illumination with a chopped laser light, and the two virtual $I-V$ curves, which correspond to those under dark (blue) and under light (red) conditions, are simultaneously obtained. The SPV spectra (green circles) are obtained by calculating the lateral shift of the two $I-V$ curves with respect to the bias voltage for the $I-V$ curve under dark condition. The central part of the SPV spectra with a tunneling current below $3 \mathrm{pA}$
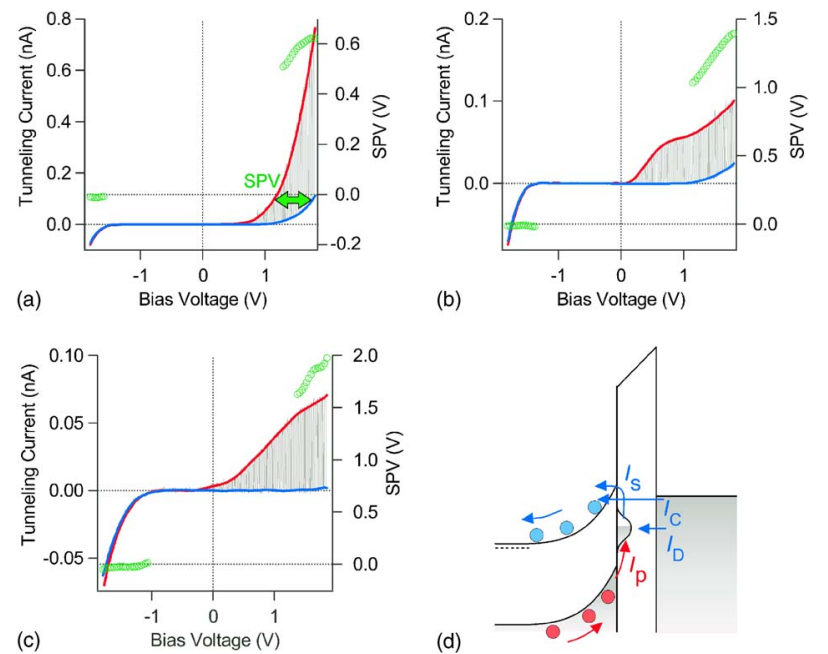

FIG. 2. (Color online) $I-V$ spectra obtained by LM-STS for (a) A and (b) B (Ga vacancy) in Fig. 1(a) and 1(c) C in Fig. 1(b) ([110] step). Red and blue curves correspond to the $I-V$ curves with and without illumination, respectively. Oscillation between the two curves is due to on and off switching of the illumination. Green dots indicate SPV determined from the shift of the two $I-V$ curves as related to the bias voltage under dark condition. (d) Schematic models for DIGS-mediated tunneling processes: $I_{s}$, current produced by thermoionic process; $I_{p}$, current of photoinduced holes; $I_{G}$, tunneling mediated by DIGS from STM tip; $I_{D}$, direct tunnel current from STM tip

changed. Therefore, the observed bias-independent profile is
to sample conduction band.
Downloaded 14 Apr 2008 to 130.158.56.186. Redistribution subject to AIP license or copyright; see http://apl.aip.org/apl/copyright.jsp is missing due to the difficulty in calculating the shift of the two $I$ - $V$ curves in that region.

As shown in Fig. 2(b), the $I$ - $V$ curve measured in a Gavacancy defect [B in Fig. 1(a)] has a hump at around 0.7 V, which is completely different from that in Fig. 2(a) and that under dark condition in Fig. 2(b). The $I-V$ curve is divided into the following three regions: (1) rapid increase $\left(V_{s}\right.$ $<0.7 \mathrm{~V})$, (2) plateau $\left(0.7 \mathrm{~V}<V_{s}<1.2 \mathrm{~V}\right)$, and (3) increase with a slope similar to that for the $I-V$ curve under dark condition $\left(1.2 \mathrm{~V}<V_{s}\right)$. What causes the difference?

SPV analysis is, in general, based on the assumption that the lateral shift of $I-V$ curves obtained under dark and light conditions is equal to the difference in the surface potential under the two conditions. This assumption is valid when a tunneling current is produced by majority carriers, corresponding to direct tunneling from the STM tip into the conduction band [Fig. 2(a)]. This condition was valid even above the atom next to the defect. However, the situation significantly changed when the $I-V$ curve was measured immediately above a Ga-vacancy defect with a DIGS. ${ }^{10,13}$

Figure 2(d) shows schematics for explaining the mechanism of the DIGS-mediated tunneling process. ${ }^{14}$ Under the dark condition, since the Fermi level is pinned by DIGS, which results in the formation of a Schottky barrier, the carrier flow is suppressed in the low-positive-sample-biasvoltage region. In contrast, under the light condition, the pinning is reduced and the minority carrier flow via DIGS $\left(I_{D}\right)$ occurs and produces tunneling current $\left(I_{t}\right)$. The tunneling current via DIGS is produced through a thermoionic process $\left(I_{s}\right)$ and the recombination of photoinduced holes $\left(I_{p}\right)$ with the electrons from the STM tip. Therefore, total tunnel current is represented as $I_{t}=I_{D}+I_{C}\left(I_{D}=I_{p}+I_{s}\right)$, where $I_{C}$ is the direct tunnel current from the STM tip to the sample conduction band. In this case, $I_{s}$ can be neglected and $I_{D}=I_{p}$.

With an increase in bias voltage, the tunneling current via DIGS increases depending on the relationship between the energy level of DIGS and the Fermi level of the tip; the first increase in $I_{t}\left(=I_{D}\right)$ depends on $V_{s}\left(V_{s}<0.7 \mathrm{~V}\right)$. At a certain bias voltage, $I_{p}$ reaches a maximum that is limited by the amount of photoinduced carriers, yielding a plateau $\left(0.7 \mathrm{~V}<V_{s}<1.2 \mathrm{~V}\right)$. For higher bias voltages $\left(1.2 \mathrm{~V}<V_{s}\right)$, direct tunneling from the STM tip to the sample conduction band occurs, which dominantly increases $I_{t}$. This is the first demonstration of the DIGS-mediated tunneling process under photoillumination observed for the isolated atomic defect.

Next, we focus on the spatial variation of SPV. Figures 3(a) and 3(b) show SPV images taken when the topographic images shown in Fig. $1\left(V_{s}=+1.7 \mathrm{~V}\right)$ are obtained. The SPV images are obtained from the $I-V$ curves acquired at equally spaced points [(a) $17 \times 17$ points, (b) $40 \times 20$ points] during the topographic imaging. Figures 3(c) and 3(d) respectively represent the cross-sectional profiles of the SPV images along line I in Fig. 3(a) and line II in Fig. 3(b), obtained under various bias voltages. Since SPV is accompanied by bias-dependent TIBB,${ }^{11-14}$ the bias-dependent component is considered to be the change in TIBB. To clarify this point, the SPV profiles after offset adjustment of those in Figs. 3(c) and 3(d) are shown in Figs. 3(e) and 3(f), respectively. The magnitude of SPV increases with bias voltage; however, the spatial variation of the cross-sectional profile remains unchanged. Therefore, the observed bias-independent profile is AlP license or copyright; see http://apl.aip.org/apl/copyright.jsp 

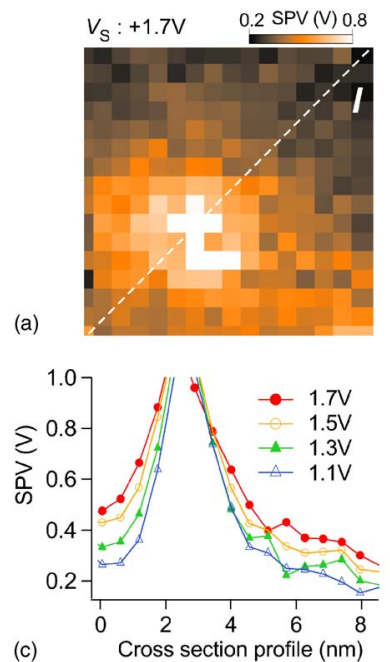

(b)
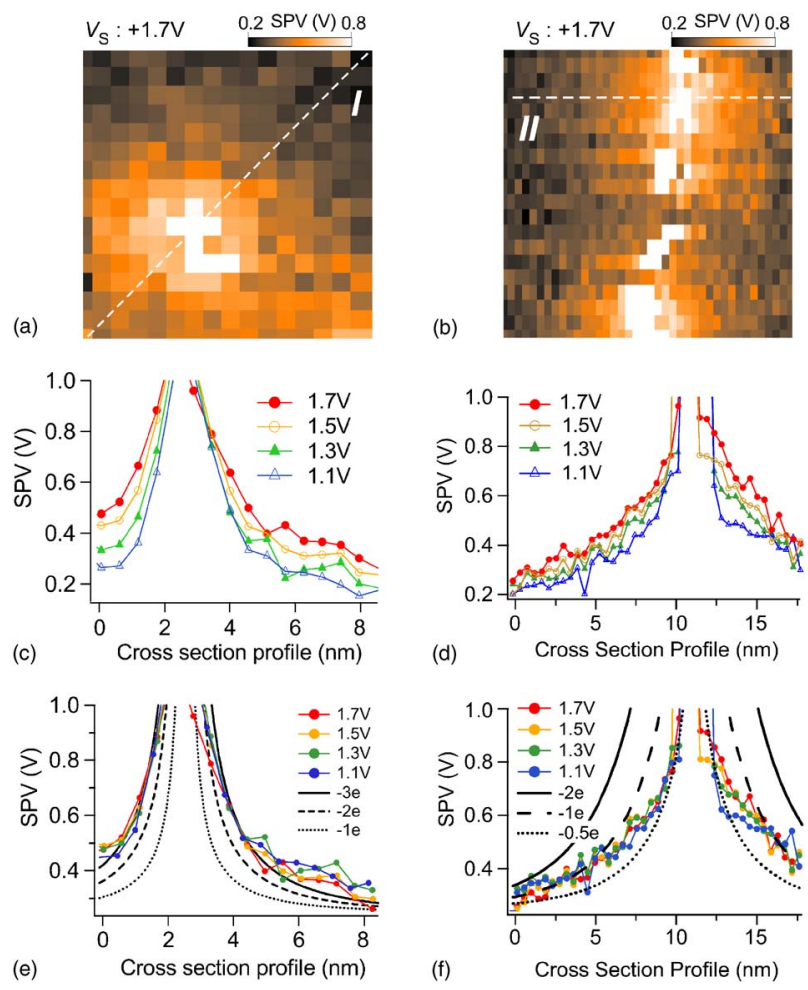

FIG. 3. (Color online) [(a) and (b)] SPV images simultaneously obtained with the topographic images shown in Fig. $1\left[V_{s}=+1.7 \mathrm{~V}\right.$; (a) $17 \times 17$ points, (b) $40 \times 20$ points]. [(c) and (d)] Cross sections of SPV mappings, obtained under various bias voltages, along line I in (a) and line II in (b), respectively. [(e) and (f)] SPV profiles in (c) and (d) after offset adjustment. Potential profiles obtained from theoretical calculations for different charges are shown together.

attributed to the intrinsic electrostatic potential variations around the defects; this the first result that directly shows the charge state of an isolated Ga vacancy.

An electrostatic potential variation around a point charge on a semiconductor surface is described with a SCP as

$$
\phi(r)=\frac{2 n e}{4 \pi \varepsilon_{0} \varepsilon_{s} r} \exp \left(-\frac{r}{R_{b}}\right),
$$

where $r$ is the lateral distance from the center of the defect and $n$ is the effective charge of the defect. $R_{b}$ is the Debye screening length of bulk $n$-GaAs. In this form, the effective charge is double counted compared with the bulk SCP because of reduction in the screening efficiency by half on the surface, where the relative dielectric constant of bulk GaAs is used. ${ }^{15}$ The Debye screening length $R_{b}$ due to the conduction of electrons is calculated to be $4.4 \mathrm{~nm}$ for the sample with a doping concentration of $2.0 \times 10^{18} \mathrm{~cm}^{-3} .{ }^{16}$ Despite considerable theoretical and experimental studies, the charge state of a Ga vacancy is still unclarified; thus, it is an issue in this case. Theoretical calculations ${ }^{17}$ and a recent positron annihilation experiment ${ }^{18}$ predict the charge of a Ga vacancy as $-3 e$, while diffusion experiments support the values for the $-2 e$ (Ref. 19) and $-1 e$ (Ref. 20) cases (e, elementary charge). According to the measurements of Si donor-Ga va- cancy pairs, the charge state at a surface is $-1 e .^{3}$ To determine the charge state of a Ga vacancy, the SCP theoretical profiles obtained for the charges of $-1 e$ to $-3 e$ are shown in Fig. 3(e). Taking account of the effect of TIBB, the value obtained by averaging the TIBB measured at several places free from defects, $0.25 \mathrm{~V}$ under $V_{s}=+1.7 \mathrm{~V}$, was added as an offset to the theoretical values. Although there is a deviation near the Ga vacancy due to the saturation of band bending in real experimental measurements, the best fitting looks achieved for the $-3 e$ state rather than the $-1 e$ state. For further advance, structure-dependent calculations are needed.

The charge distribution for the step edge [Fig. 3(d)] is wider than that for the Ga vacancy [Fig. 3(c)], which is attributed to the fact that the step edge consists of continuously ordered point defects equally spaced along the step. The potential distributions, calculated by the superposition of the aligned point charges for $-1 e$ per double lattice spacing (represented by $-0.5 e)$ and $-1 e$ and $-2 e$ per lattice spacing, ${ }^{6}$ are shown in Fig. 3(d). The SPV profile is close to the distribution calculated for the $-1 e$ state with this simple model.

In conclusion, SCPs induced around a charged Ga vacancy and a step edge were visualized on the nanometer level. Furthermore, the charge states of the individual defects were determined at the atomic level.

We thank Ms. Rie Yamashita, a member of our group, for her help in preparing this paper.

${ }^{1}$ E. G. Seebauer and M. C. Kratzer, Mater. Sci. Eng., R. 55, 57 (2006).

${ }^{2}$ Ph. Ebert, Surf. Sci. Rep. 33, 121 (1999).

${ }^{3}$ C. Domke, Ph. Ebert, M. Heinrich, and K. Urban, Phys. Rev. B 54, 10288 (1996).

${ }^{4}$ J. Gebauer, E. R. Weber, N. D. Jäger, K. Urban, and Ph. Ebert, Appl. Phys. Lett. 82, 2059 (2003).

${ }^{5}$ Ph. Ebert, X. Chen, M. Heinrich, M. Simon, K. Urban, and M. G. Lagally, Phys. Rev. Lett. 76, 2089 (1996).

${ }^{6}$ M. Heinrich, C. Domke, Ph. Ebert, and K. Urban, Phys. Rev. B 53, 10894 (1996).

${ }^{7}$ C. Domke, Ph. Ebert, M. Heinrich, and K. Urban, J. Vac. Sci. Technol. B 16, 2825 (1998).

${ }^{8}$ L. Kronik and Y. Shapira, Surf. Sci. Rep. 37, 1 (1999).

${ }^{9}$ M. Berthe, S. Yoshida, Y. Ebine, K. Kanazawa, A. Okada, A. Taninaka, O Takeuchi, N. Fukui, H. Shinohara, S. Suzuki, K. Sumitomo, Y. Kobayashi, B. Grandidier, D. Stievenard, and H. Shigekawa, Nano Lett. 7, 3623 (2007).

${ }^{10}$ S. Grafstrom, J. Appl. Phys. 91, 1717 (2002).

${ }^{11}$ O. Takeuchi, S. Yoshida, and H. Shigekawa, Appl. Phys. Lett. 84, 3645 (2004).

${ }^{12}$ S. Yoshida, Y. Kanitani, R. Oshima, Y. Okada, O. Takeuchi, and H. Shigekawa, Phys. Rev. Lett. 98, 026802 (2007).

${ }^{13}$ M. W. J. Prins, R. Jansen, R. Groeneveld, A. Gelder, and H. Kempen, Phys. Rev. B 53, 8090 (1996).

${ }^{14}$ M. McEllistrem, G. Haase, D. Chen, and R. J. Hamers, Phys. Rev. B 70, 2471 (1993).

${ }^{15}$ M. Kremar, W. M. Saslow, and M. B. Weimer, Phys. Rev. B 61, 13821 (2000).

${ }^{16}$ R. B. Dingle, Philos. Mag. 46, 831 (1955).

${ }^{17}$ S. B. Zhang and J. E. Northrup, Phys. Rev. Lett. 67, 2339 (1991).

${ }^{18}$ J. Gebauer, M. Lausmann, F. Redmann, R. Krause-Rehberg, H. S. Leipner, E. R. Weber, and Ph. Ebert, Phys. Rev. B 67, 235207 (2003).

${ }^{19}$ H. Bracht, M. Norseng, E. E. Haller, K. Eberl, and M. Cardona, Solid State Commun. 112, 301 (1999).

${ }^{20}$ R. M. Cohen, Mater. Sci. Eng., R. 20, 167 (1997). 